\title{
Assessment of CaMV-mediated gene silencing and integration of CaMV into GM plants with a 35S RNA promoter
}

\author{
Julie SQUIRES ${ }^{1}$, Jennifer STEPHENS ${ }^{1}$, James E. SCHOELZ² and Peter PALUKAITIS ${ }^{1 *}$ \\ 1 Scottish Crop Research Institute, Invergowrie, Dundee DD2 5DA, UK \\ 2 Div. of Plant Sciences, University of Missouri, Columbia, MO 65211, USA
}

Four GM plant species (Arabidopsis thaliana, Brassica napus, Nicotiana benthamiana and N. tabacum), each expressing the gene encoding the jellyfish green fluorescent protein (GFP) regulated by the cauliflower mosaic virus (CaMV) 35S RNA promoter, were assessed for the extent of transgene silencing and viral genome integration following infection by CaMV. The first three species are systemic hosts of CaMV, while $\mathrm{N}$. tabacum is only a local host for a few strains of CaMV. A generalized systemic silencing of the GFP transgene was not observed in a total of 100 plants of each species infected with CaMV, although some localized loss of GFP was observed in CaMV-infected $\mathbf{N}$. benthamiana leaves, and some loss of fluorescence was observed in older leaves of uninfected as well as infected plants. Progeny seedlings obtained from the above infected plants also did not exhibit transgene silencing showing that virus infection did not affect the stability of the transgene. These progeny plants also did not show signs of virus infection, indicating that the presence of the CaMV 35S RNA promoter sequences in the plant genome did not potentiate seed transmission of the virus. Integration of infective CaMV into the CaMV 35S RNA promoter could not be detected in 944 samples taken from leaves of the above infected plant species or in 2912 samples taken from progeny seedlings. Based on a detection limit of one copy per 4000 haploid genomes, we conclude that if integration of virus does occur into the CaMV 35S RNA promoter, then it occurs at such a low frequency as to be insignificant.

Keywords: cauliflower mosaic virus / 35S promoter / integration / recombination / transgene silencing

\section{INTRODUCTION}

Most of the GM plants found in the fields of the various countries propagating them contain transgenes regulated in their expression by the 35S RNA promoter of cauliflower mosaic virus (CaMV). This is also true of many GM plants still under development in various laboratories. This viral promoter functions constitutively in all tissues tested and is one of the most active promoters found in plants (Jefferson et al., 1987). While plant gene promoters that are inducible and/or tissue specific have been described, and some of these have been used for gene expression in transgenic plants, the CaMV $35 \mathrm{~S}$ RNA promoter is still the workhorse of choice for the expression of many transgenes. However, some concerns have been raised about the use of the CaMV 35S RNA promoter and possible risks it may pose to human health and to the environment (Ho et al., 1999). The concerns were responded to (Hull et al., 2000; Morel and Tepfer,

\footnotetext{
*Corresponding author: Peter.Palukaitis@ @cri.ac.uk
}

2000) and the responses were received with renewed criticism (Ho et al., 2000). Therefore, some of the issues raised have been assessed by additional experimentation. The CaMV 35S RNA promoter has been assessed for its ability to function in mammalian cells (Tepfer et al., 2004; Vlasák et al., 2003), as was done previously in bacteria (Assaad and Signer, 1990), yeast (Pobjecky et al., 1990; Rüth et al., 1992; 1994), fungi (Sun et al., 2002) and toad eggs (Ballas et al., 1989). Nevertheless, while the CaMV 35S RNA promoter is active to different extents in these various organisms, to date there is still no evidence supporting the scenario envisioned by Ho et al. $(1999 ; 2000)$ for the risks to human or animal health by using the CaMV 35S RNA promoter in GM plants. However, two other questions raised by Ho and colleagues (1999) have not been adequately investigated experimentally. They questioned whether the CaMV 35S RNA promoter functions as a hot spot for integration of infective CaMV into plant genomes, and whether infective CaMV could have an effect on the expression and/or stability of transgenes regulated by the 35S RNA promoter. 


\section{J. Squires et al.}

Studies examining the effects of infection of transgenic oilseed rape (OSR; Brassica napus) by CaMV have shown that the transgene expression can be inhibited late during infection by both transcriptional gene silencing and post-transcriptional gene silencing, depending on the absence or presence, respectively, of CaMV transcription regulation sequences (Al-Kaff et al., 1998). This silencing occurred in OSR at the same time as recovery from infection became apparent. Furthermore, whether transgene silencing occurred and the extent of transgene silencing were shown to depend upon the strain of CaMV used and at what time infection occurred during the growth cycle of the plants, respectively (Al-Kaff et al., 2000). However, it is not known to what extent such transgene silencing would occur in other plant species that are susceptible to CaMV, especially those unlike OSR, where recovery from infection does not occur, or whether a strain of CaMV that does not silence transgene expression in OSR also does not do so in other plant species. Moreover, transgenic plant species that do not support a progressive infection by CaMV have not been evaluated for possible effects on the expression of transgenes driven by the CaMV 35S RNA promoter caused by RNA silencing mediated by CaMV infection. Therefore, this study was undertaken to examine whether infection by CaMV resulted in silencing of 35S RNA promoter-driven transgene expression in other species, and whether integration of CaMV occurred into the 35S RNA promoter during infection of GM plants that express a transgene driven by the CaMV 35S RNA promoter.

\section{RESULTS}

\section{Description of the system}

For these analyses, four species of plants were used, each expressing the green fluorescent protein (GFP) under the regulation of the CaMV 35S RNA promoter and the nos terminator. The plant species were Arabidopsis thaliana, B. napus (OSR), Nicotiana benthamiana and N. tabacum (tobacco). A. thaliana and B. napus are members of the family Brassicaceae and are hosts of CaMV. OSR is a major crop species, while A. thaliana is an experimental plant species for which the nucleotide sequence of the entire genome has been determined. $N$. benthamiana and $N$. tabacum are members of the family Solanaceae, of which only a few species are infected by a limited number of CaMV strains. However, both of these plant species also are experimental host plants that have been used for studies involving transgenic plants. In the case of tobacco, such studies have extended to the field. While $N$. benthamiana can be infected systemically by some strains of CaMV, tobacco cannot, and tobacco either shows no infection, or infection limited to the inoculated leaf.
Transgenic plants that expressed a gene encoding the GFP under the control of the CaMV 35S RNA promoter were inoculated with either CaMV strain W260 (for local infection of tobacco) (Gracia and Shepherd, 1985) or the chimeric CaMV H7 (Schoelz et al., 1986a), in which gene VI of the aphid non-transmissible strain CM 1841 had been replaced with gene VI of the solanaceousinfecting strain D4 (for infection of A. thaliana, OSR and $N$. benthamiana). Gene VI affects both host range and pathogenicity (reviewed by Schoelz, 2006). The disease induced by the chimeric CaMV strain $\mathrm{H} 7$ was generally mild in A. thaliana, OSR and N. benthamiana, although responses in individual plants varied from mild to lethal. By contrast, CaMV strain W260 did not induce any symptoms on tobacco.

\section{Assessment of transgene silencing in systemically infected plant species}

\section{Transgene silencing in OSR}

Fifty GM OSR plants expressing the GFP were infected with CaMV strain $\mathrm{H} 7$ in each of two separate experiments, and the extent of transgene expression was assessed by examining the level of GFP accumulation at 10day intervals up to 70 or 90 days post inoculation (dpi), relative to 10 mock-inoculated, control GM OSR plants. This was done by examining the level of GFP fluorescence with a binocular microscope equipped with a camera capable of quantifying the fluorescence intensity. The results from the first experiment (Fig. 1A) and the second experiment (Fig. 1B) were essentially the same; in both experiments, there was considerable variation in the fluorescence intensity between individual plants receiving the same treatment, although this level of variation was not significantly different in mock-inoculated $v s$. CaMVinfected plants. Some reduction in fluorescence of the GFP was seen in the oldest leaves but this occurred in both mock-inoculated and CaMV-infected plants (Fig. 2) and was observed previously for transgene expression driven by a CaMV 35S RNA promoter (Mitsuhara et al., 1996). The inoculated leaves of both virus-infected and mock-inoculated plants showed a decrease of green fluorescence with time (Fig. 1B). Otherwise, there was no generalized effect that could be interpreted as silencing of transgene expression observed following infection by this mild strain of $\mathrm{CaMV}$, in contrast to that reported for the severe strain Cabb B-JI (Al-Kaff et al., 1998). This is unlikely to be due to different environmental conditions, since other strains of CaMV also did not silence transgene expression, regardless of whether they were mild or severe (Al-Kaff et al., 2000).

Seeds obtained from four CaMV-infected OSR plants showing reduction in GFP fluorescence were germinated and their fluorescence again was screened. All seedlings 


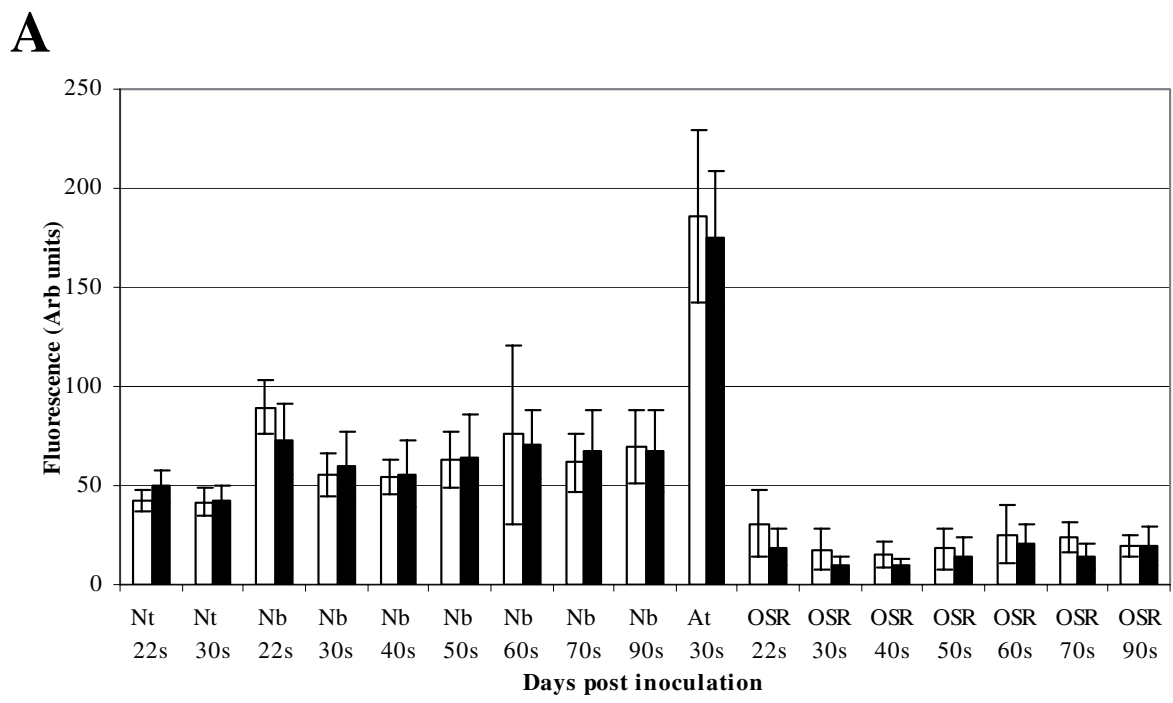

B

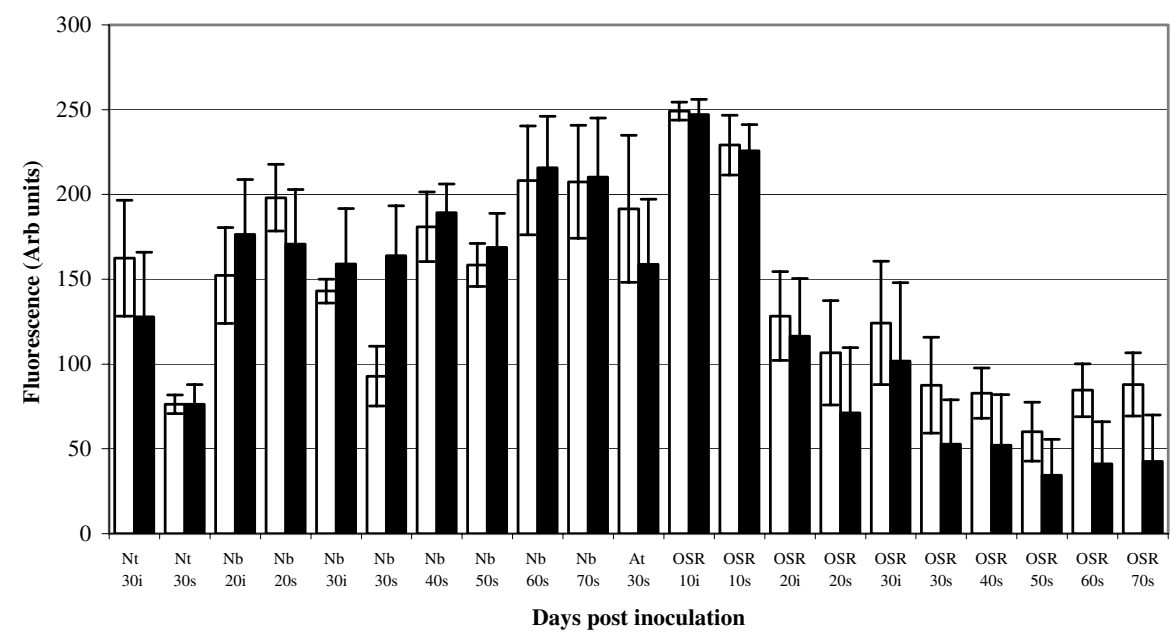

Figure 1. Mean fluorescence of CaMV 35S RNA promoted GFP plants measured at various days post inoculation under UV light at $5 \times$ magnification in two separate experiments. A. thaliana $[\mathrm{At}], N$. benthamiana $[\mathrm{Nb}]$ and $B$. napus $[\mathrm{OSR}]$ plants were inoculated with CaMV strain $\mathrm{H} 7$ and $N$. tabacum [Nt] plants were inoculated with CaMV strain W260. Mock inoculated plants are shown as empty bars. Virus inoculated plants are shown as black bars. Error bars are $+/-$ standard deviation. In the first experiment (A), only upper systemic leaves (s) were examined, while in the second experiment (B), both inoculated leaf samples (i) and upper or systemic leaf samples (s) were examined. In experiment (B), the average background fluorescence for no-GM plants was 42 for A. thaliana, and 39 for $N$. benthamiana and B. napus.

exhibited a typical profile of GFP fluorescence, and did not show any evidence supporting transgene silencing or virus infection (data not shown).

\section{Transgene silencing in A. thaliana}

Fifty A. thaliana plants were inoculated with CaMV strain $\mathrm{H} 7$ in two separate experiments and were assessed for effects on GFP accumulation at 30 dpi. Infection by CaMV strain $\mathrm{H} 7$ killed 10 of the 50 plants from the first experiment, but led to severe symptoms with production of flower stalks, flowers and siliques in most of the remaining $40 \mathrm{CaMV}$-infected plants (Fig. 3). In two of these plants, reduction of GFP expression was observed in the rosette and cauline leaves and flower stalks (data not shown). For the surviving plants, there was no other difference observed in fluorescence recorded with 


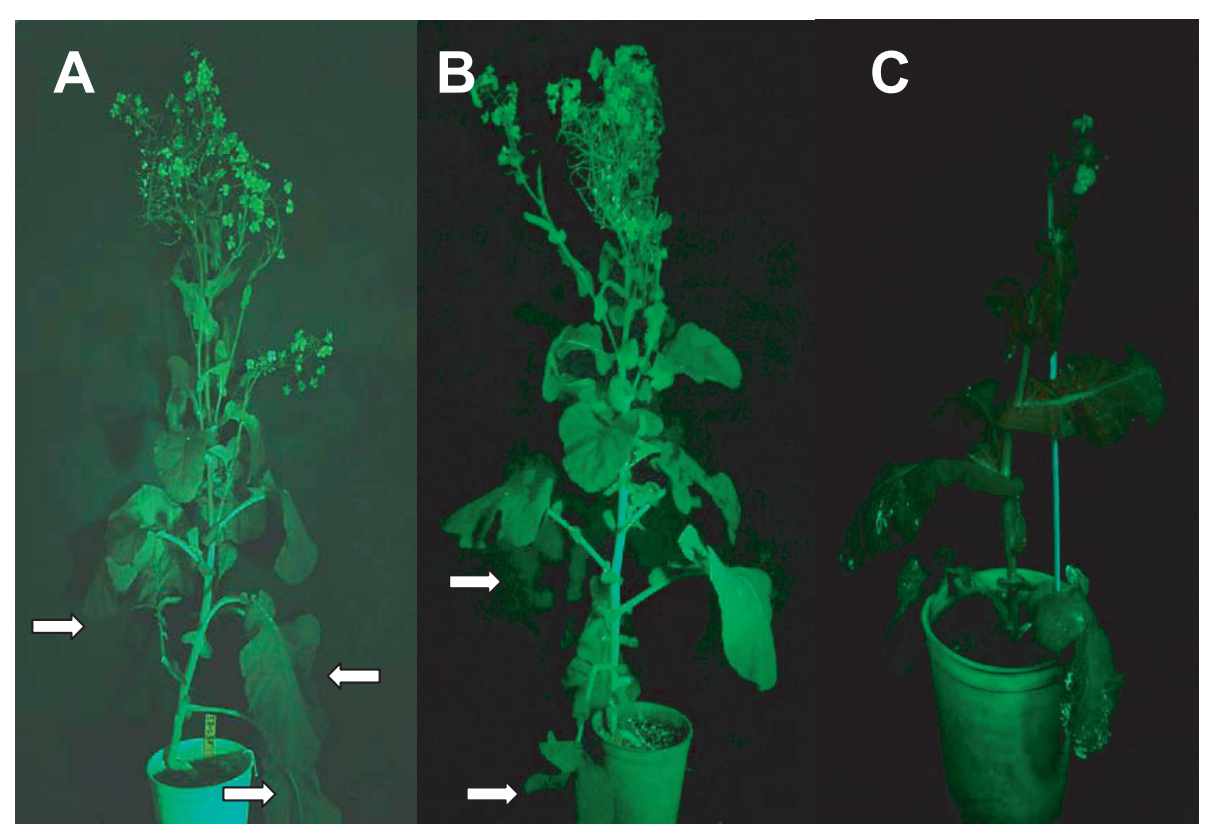

Figure 2. B. napus [oilseed rape] expressing 35S-driven GFP, 50 days post inoculation with CaMV H7 (A) or mock inoculated (B). Both plants showed silencing (lack of green fluorescence) only in the lower regions of the plant (arrows). (C) Non-GM oilseed rape plants. All plants were photographed under UV light using a Kodak Wratten 58 Tricolor Green Filter.

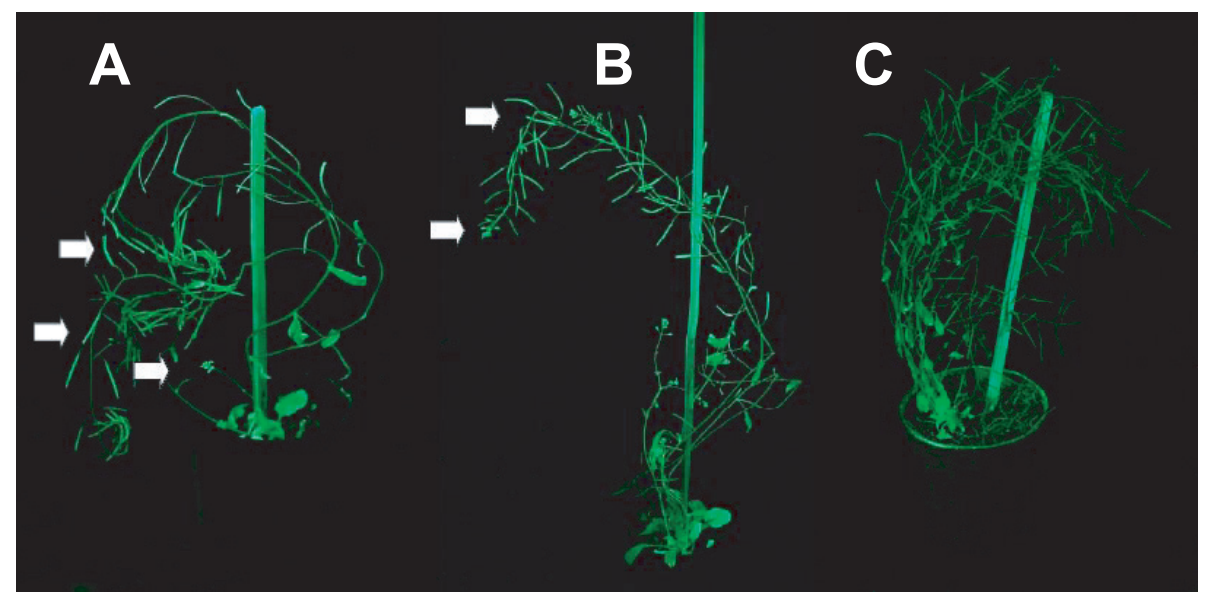

Figure 3. Arabidopsis thaliana expressing 35S-driven GFP. Plants were photographed under UV light using a Kodak Wratten 58 Tricolor Green Filter, 30 days post inoculation with CaMV H7 (B) or mock inoculated (A). Lower regions of both plants showed GFP silencing, with siliques and new flower initials glowing (arrows), as compared with non-GM A. thaliana plants (C).

the binocular microscope and camera, but there was individual plant variation in the level of fluorescence (Fig. 1). Loss of fluorescence was observed in the lower parts of the plants, but this was seen in both mock-inoculated and virus-infected plants (Fig. 3). These plants were assessed for suppression of transgene mRNA expression $v s$. possible modification or loss of the transgene, by examining expression of the transgene in the next generation.
Seeds (10) obtained from the first experiment of all the surviving $A$. thaliana plants were sown and germinated and the seedlings were screened for fluorescence. All showed similar fluorescence, indicating that any reduced fluorescence observed was not carried through to the next generation. This indicates that any such effect was transient in the infected population, and was not affecting the stability of the transgene in subsequent generations. 
In the second experiment, infection by $\mathrm{CaMV}$ strain $\mathrm{H} 7$ killed eight of the 50 plants, but led to severe symptoms with production of flower stalks, flowers and siliques in most of the remaining $42 \mathrm{CaMV}$-infected plants. In one of these latter plants, loss of GFP accumulation was observed in the rosette and cauline leaves and flower stalks, with only the basal root expressing GFP (data not shown). Two of these plants showed atypical growth forms, one being extremely stunted, with very hairy leaves, while the second plant exhibited thickened stems and delayed flowering, typical of the effect of the clavata gene (not shown). For the remaining 40 plants, there was no other difference observed in fluorescence recorded with the binocular microscope and camera, but there was individual plant variation (Fig. 1). Loss of fluorescence also was observed in the lower parts of the plants, although again this was seen in both mockinoculated and virus-infected plants.

These second experiment plants were assessed for suppression of transgene mRNA expression $v s$. potential loss or modification of the transgene, by examining expression of the transgene in the next generation. Fifteen A. thaliana seedlings of each of the forty plants were germinated and followed through the various stages of plant development. The progeny plants developed normally and showed GFP fluorescence, seed set and growth (data not presented).

In the case of the two A. thaliana plants that had abnormal development after infection with CaMV (out of 50 infected plants), the plant that was extremely stunted with hairy leaves did not set seed. However, the plant with a thickened stem and delayed flowering set seed that germinated normally and produced normal plants that also expressed (35S-driven) GFP. Therefore, the phenotype observed in the parental plants was a manifestation of viral infection and not due to any permanent effect on the plant genome.

\section{Transgene silencing in $\mathrm{N}$. benthamiana}

Fifty GFP-expressing, GM $N$. benthamiana plants were infected with $\mathrm{CaMV}$ strain $\mathrm{H} 7$ in two separate experiments, and the extent of GFP accumulation was assessed at 10-day intervals up to 70 or $90 \mathrm{dpi}$, relative to 10 mock-inoculated, control GM $N$. benthamiana plants. As in the other host species above, there was variation in the extent of fluorescence between different plants undergoing the same treatment, whether they were infected or not (Fig. 1). While there was no evidence for general suppression of GFP expression, loss of fluorescence again was observed in the oldest leaves of older plants, and again this occurred in both mock-inoculated (not shown) and CaMV-infected plants (Fig. 4A). However, limited, localized loss of green fluorescence also was observed in upper leaves of plants systemically infected with CaMV, but not in the upper leaves of mockinoculated plants. This was manifested in loss of fluorescence occurring in chlorotic lesions formed in response to infection by CaMV (Fig. 4B). The loss of fluorescence did not spread beyond these lesions to the adjacent tissues, and therefore did not affect the recorded fluorescence quantification (Fig. 1). It is not know why these chlorotic lesions showed loss of GFP accumulation. The observation was not investigated further.

\section{Assessment of transgene silencing in locally infected tobacco}

Most of the GM plant species generated to date that express genes under the regulation of the CaMV 35S RNA promoter are not systemic hosts of CaMV. Many transgenes have been expressed in tobacco, which is resistant to systemic infection by CaMV, and also resistant to local infection by most strains of CaMV (Goldberg et al., 1991; Gracia and Shepherd, 1985). Therefore, we used CaMV strain W260, as this was capable of limited local infection in tobacco, to examine the effect that local CaMV infection might have on the local and systemic expression of a transgene regulated by the CaMV 35S RNA promoter. In two independent experiments, 50 GFP-expressing GM tobacco plants were infected with CaMV strain W260, and the extent of GFP accumulation was assessed at $30 \mathrm{dpi}$, relative to GFP accumulation in non-infected plants (Fig. 1). In the second experiment, the effect on GFP accumulation also was assessed in non-infected upper leaves of the inoculated tobacco plants (Fig. 1B). Inoculated leaves could not be assessed much beyond $30 \mathrm{dpi}$, since they then became senescent. Some CaMV-infected plants did show a slight reduction in fluorescence, but no significant difference in GFP accumulation was observed in either the inoculated leaves or the non-infected upper leaves of most plants (Fig. 1). Seeds taken from six selected plants showing a slight reduction in GFP accumulation were germinated. The progeny plants did not show loss of GFP expression, but did show a slight reduction in fluorescence, indicating that they were accumulating slightly less GFP. Therefore, the above result is likely to be a consequence of either individual or somaclonal variation in expression of GFP.

\section{Assessment of viral integration into the CaMV 35S RNA promoter in GM plants}

Although a generalized effect on GFP transgene expression was not observed here, lower levels of fluorescence 


\section{J. Squires et al.}
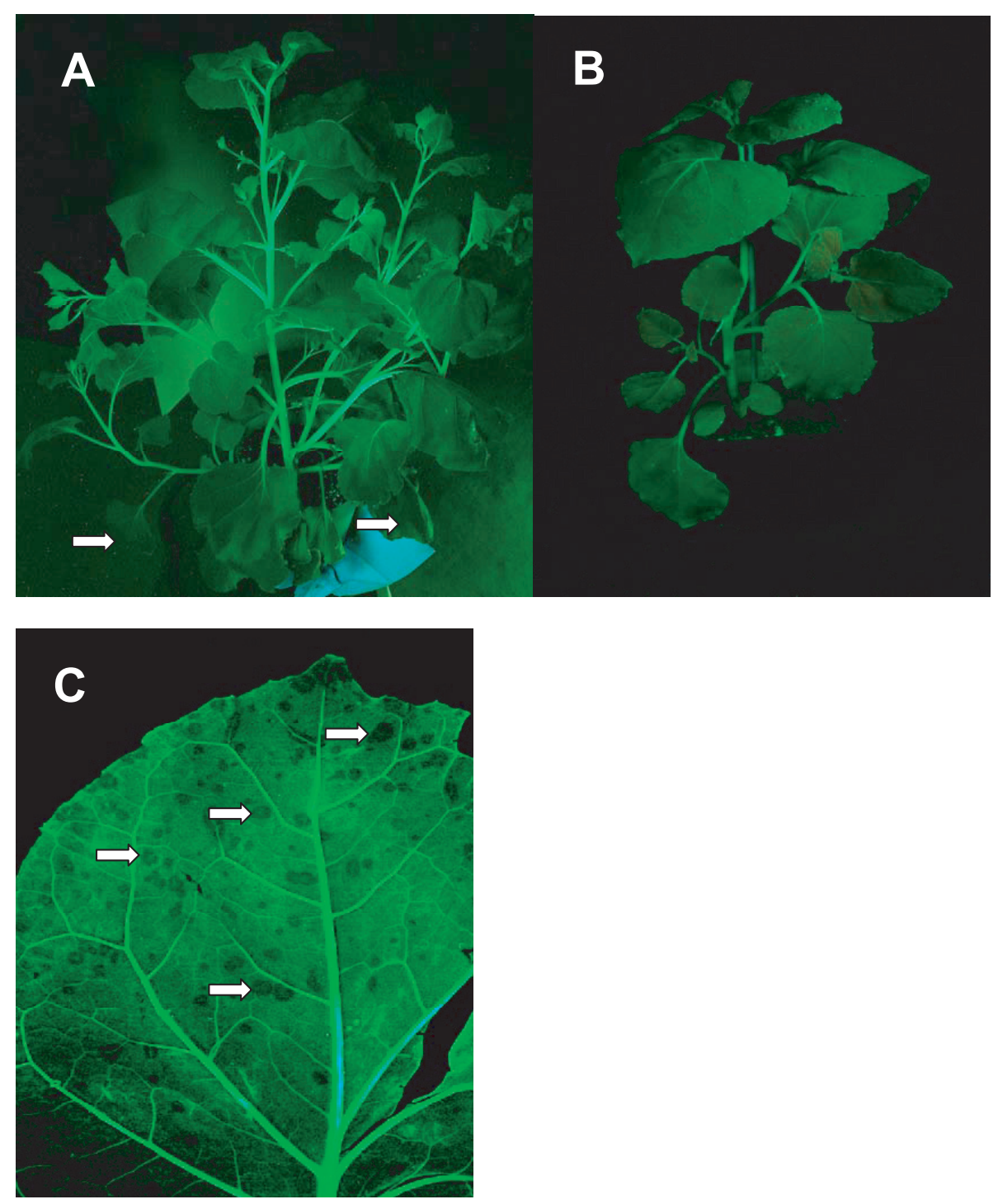

Figure 4. $N$. benthamiana expressing 35S-driven GFP. (A) A 35S-GFP GM plant showing fluorescence in the upper leaves and stems, but silencing of GFP expression in the lower leaves of the plant (arrows), 50 days post inoculation with CaMV H7. (B) A noninfected, non-GFP plant. (C) Close up of a leaf showing GFP silenced regions across the leaf at sites of chlorotic lesions (arrows), 90 days post inoculation with CaMV H7. Plants were viewed and photographed under UV light using a Kodak Wratten 58 Tricolor Green Filter.

seen in some plants may have been a consequence of either plant responses to virus infection or integration of the virus into the homologous CaMV 35S RNA promoter sequence in some, but clearly not most cells of the infected plants. Before examining whether CaMV could integrate into the genome of GM plants (whether hosts or non-hosts of CaMV), we first had to establish a detection endpoint for such putative integration. Therefore, decreasing amounts of 35S-GFP OSR tissues either alone, or mixed with a constant amount of non-GM OSR tissues were extracted, and the DNAs were subjected to the PCR using primers designed to detect the CaMV 35S RNA promoter. Our extraction and PCR methods were sensitive enough to detect $0.1 \mathrm{mg}$ of tissue containing the CaMV 35S RNA promoter in a total of $200 \mathrm{mg}$ of plant tissues (Fig. 5). That is, we have the capacity to detect the equivalent of one cell containing integrated sequences in 2000 cells (or the equivalent of one copy per 4000 haploid 


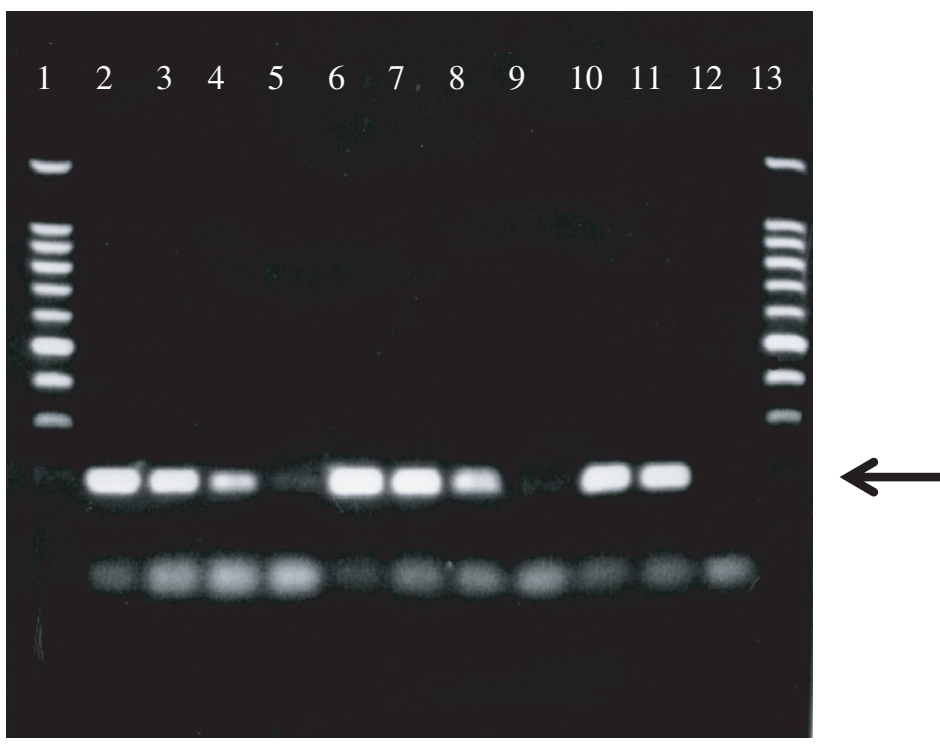

Figure 5. Analysis of dilution endpoint for detection of CaMV 35S RNA promoter sequences. The 35S RNA promoter PCR products $(5 \mu \mathrm{L})$ were analyzed after 40 cycles on a $2 \%$ agarose gel run at $70 \mathrm{~V}$ for $30 \mathrm{~min}$. Samples: (1) M = molecular weight markers; $(2-$ 11) DNAs obtained from the following tissues: (2) $100 \mathrm{mg}$ (NonGFP) plus $100 \mathrm{mg}$ (GFP); (3) $190 \mathrm{mg}$ (NonGFP) : $10 \mathrm{mg}$ (GFP); (4) $199 \mathrm{mg}$ (NonGFP) : $1 \mathrm{mg}$ (GFP); (5) $199.9 \mathrm{mg}$ (NonGFP) : $0.1 \mathrm{mg}$ (GFP); (6) $100 \mathrm{mg}$ (GFP); (7) $10 \mathrm{mg}$ (GFP); (8) $1 \mathrm{mg}$ (GFP); (9) $0.1 \mathrm{mg}$ (GFP); (10) chloroform only extraction (GFP); (11) phenol/chloroform extraction (GFP); (12) no template control; (13) $\mathrm{M}=$ Promega $100 \mathrm{bp}$ markers. Arrow = expected band of $194 \mathrm{bp}$.

genome copies). No such PCR products were obtained from CaMV-infected plants or non-GM plants of all four species (data not shown).

The GM plants that then were assessed for integration of CaMV into the CaMV 35S RNA promoter were A. thaliana, OSR, $N$. benthamiana and tobacco, all expressing the gene encoding the GFP under the control of the CaMV 35S RNA promoter. DNAs were extracted from infected plants of each species, and were assessed for integration at 30 days after inoculation of A. thaliana (10 plants) and tobacco (10 plants), and at 30 , 60 and 90 days after inoculation of OSR (10 plants) and $N$. benthamiana (10 plants). Both the directly-infected GM plants and progeny seedlings of the infected GM plants were analyzed. The PCR was done using primers designed to bind to gene VI of CaMV (as the forward primer), and to a site within the GFP gene in the plant DNA (as the reverse primer) (Fig. 6).

This primer combination did not amplify DNA using either purified CaMV DNA, or DNA extracted from non-infected GM or non-GM plants of the four species as templates (data not shown), but was able to amplify a segment of DNA from CaMV-GFP, a virus with gene II replaced by the GFP gene (Fig. 7). The same primer combination did not yield PCR products from the 944 samples of CaMV-infected plants or the 2912 progeny seedlings (Tab. 1). That is, this sensitive assay did not provide any evidence that integration of $\mathrm{CaMV}$ into the CaMV
35S RNA promoter had occurred in the 3856 samples examined, indicating that either integration had not occurred or that it occurred at a level below the sensitivity of our system (i.e., one copy per 4000 haploid genomes).

Since the extent of recombination leading to integration would be heavily influenced by the degree of sequence identity between the virus and the CaMV sequences present in the 35S RNA promoters, we examined the level of sequence identity between the viruses used here and the CaMV sequences present in the transgene promoters. All four transgenic species were transformed with T-DNAs that originally derived from the cassette of the 35S-GFP gene generated by Haseloff et al. (1997) in the transformation vector pBI121 (Jefferson et al., 1987), replacing the GUS gene with the GFP gene. The pBI121 vector was a modification of the vector $\mathrm{pBIN} 19$ containing the CaMV sequences including the 35S RNA promoter, described by Bevan et al. (1985), as modified further by Baulcombe et al. (1986). Thus, pBI121 contained 948 bp derived from CaMV (sequences 64947441). The transcription initiation start site is located at position 7435 (Guilley et al., 1982) and the core promoter is located within $46 \mathrm{bp}$ upstream of the transcription initiation site, but transcription enhancer sequences are located between 89 and 148 bp upstream of the transcription initiation site (Odell et al., 1985; Ow et al., 1987). Thus, the transformation vectors contained an additional $\sim 790$ bp of CaMV sequences that were not necessary for 


\section{J. Squires et al.}

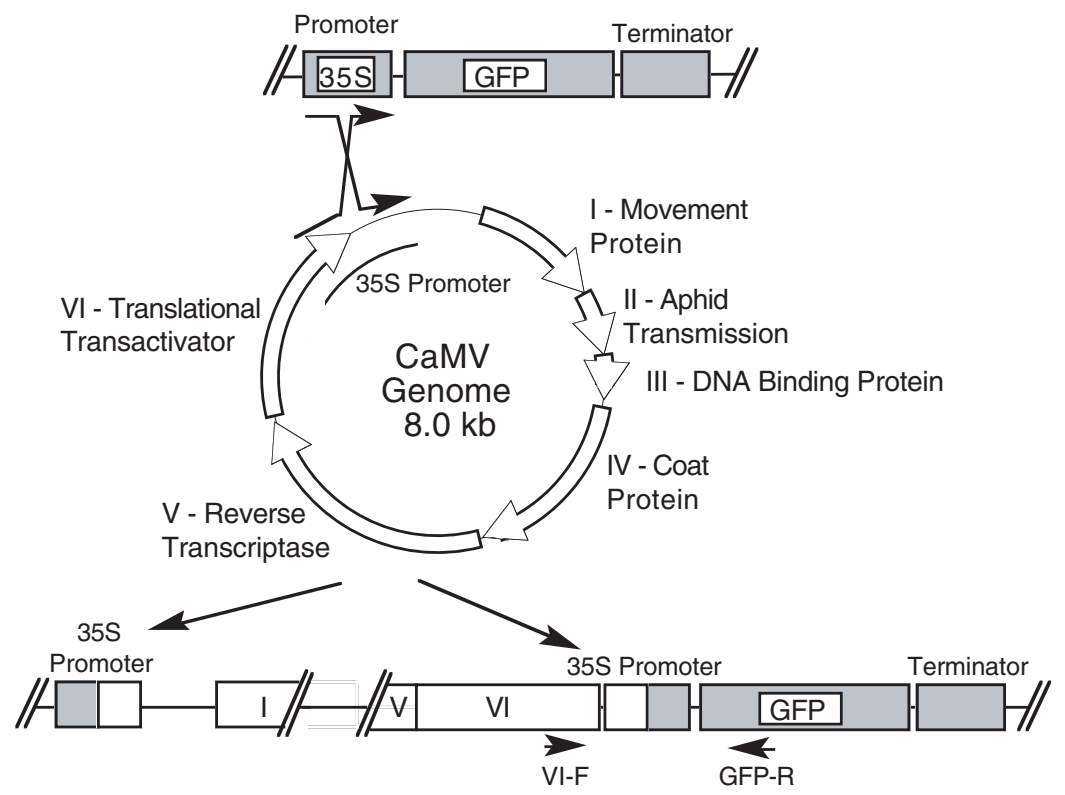

Figure 6. Detection of a putative recombinant between CaMV DNA and the CaMV 35S RNA promoter in transgenic plants. The figure illustrates the outcome of a single homologous crossover event between CaMV DNA and the CaMV 35S RNA promoter. Transgene sequences are represented by grey boxes, whereas the viral genes are illustrated by open boxes. The viral genome map details the six CaMV genes that encode protein products and the position of the 35S RNA promoter. A putative recombinant would be detected by the PCR using the forward primer within CaMV Gene VI (VI-F) and the reverse primer in the GFP transgene (GFP-R).

Table 1. Number of samples examined for potential integration of CaMV into the genome of 35S RNA promoted GFP plants.

\begin{tabular}{lccccc}
\hline \hline Species $^{1}$ & Inoculated leaves & \multicolumn{3}{c}{ Systemic leaves } & \multirow{2}{*}{ Progeny seedlings } \\
& & $30 \mathrm{dpi}$ & $60 \mathrm{dpi}$ & $90 \mathrm{dpi}$ & \\
\hline A. thaliana $_{\text {B. } \text { napus }^{2}}$ & 80 & 80 & $\mathrm{NS}$ & $\mathrm{NS}$ & 800 \\
N. benthamiana $^{3}$ & 80 & 80 & 80 & 80 & 592 \\
N. tabacum $^{*}$ & 80 & 80 & 72 & 72 & 720 \\
\hline
\end{tabular}

${ }^{1}$ Sampled: ten parents of each species, up to 10 seedlings per parent taken at various days post inoculation (dpi).

${ }^{2}$ B. napus had fewer seedlings for some: one with only three seedlings, one with only one seedling and one plant that did not survive to set viable seed.

${ }^{3}$ In $N$. benthamiana, one plant died after one month post inoculation.

${ }^{4} \mathrm{NS}=$ not sampled.

transcription mediated by the 35S RNA promoter. Within the $948 \mathrm{bp}$ of CaMV sequences present in the transgenic plants, derived from strain Cabb-BS (Franck et al., 1980), the levels of sequence similarities to the DNAs of CaMV strains D4 (the source of the corresponding sequences in the hybrid strain H7) and W260 were 96.0 and $96.6 \%$, respectively (Tab. 2), with the longest contiguous stretch of sequence identity being 185 and $136 \mathrm{bp}$ between strains Cabb-BS and D4, and 185 and 83 bp between strains Cabb-BS and W260. These strains and other wellcharacterized strains of CaMV (Cabb B-JI, and CM1841) varied in sequence from each other in this region by $0.8 \%$ to $5.4 \%$ (Tab. 2). Thus, the degree of sequence identity between these strains and the CaMV sequences present in the transgenic plants was quite high and sufficient to allow homologous recombination to occur.

To ensure that the samples analyzed were amplifiable, and to rule out poor quality template as the reason for not detecting any integration, a subset of samples (344) covering all four plant species, different sampling timepoints and the progeny seedlings were amplified for the presence of the integrated CaMV 35S RNA promoter and GFP gene. These data showed that the template was of amplifiable quality and quantity for all samples tested (data not presented). Therefore, despite the high level of sequence identity between the infecting CaMV genome 
CaMV-mediated transgene inactivation and integration

Table 2. Sequence identity between nucleotides 6494 and 7441 among strains of CaMV.

\begin{tabular}{lccccc}
\hline \hline \multirow{2}{*}{ Strains } & Cabb-BS & D4 & W260 & CM1841 & Cabb B-JI \\
\hline Cabb-BS & $100 \%$ & $96.0 \%$ & $96.6 \%$ & $96.0 \%$ & $95.6 \%$ \\
D4 & $96.0 \%$ & $100 \%$ & $95.4 \%$ & $99.2 \%$ & $97.2 \%$ \\
W260 & $96.6 \%$ & $95.4 \%$ & $100 \%$ & $95.4 \%$ & $94.6 \%$ \\
CM1841 & $96.0 \%$ & $99.2 \%$ & $95.4 \%$ & $100 \%$ & $97.0 \%$ \\
Cabb B-JI & $95.6 \%$ & $97.2 \%$ & $94.6 \%$ & $97.0 \%$ & $100 \%$ \\
\hline
\end{tabular}

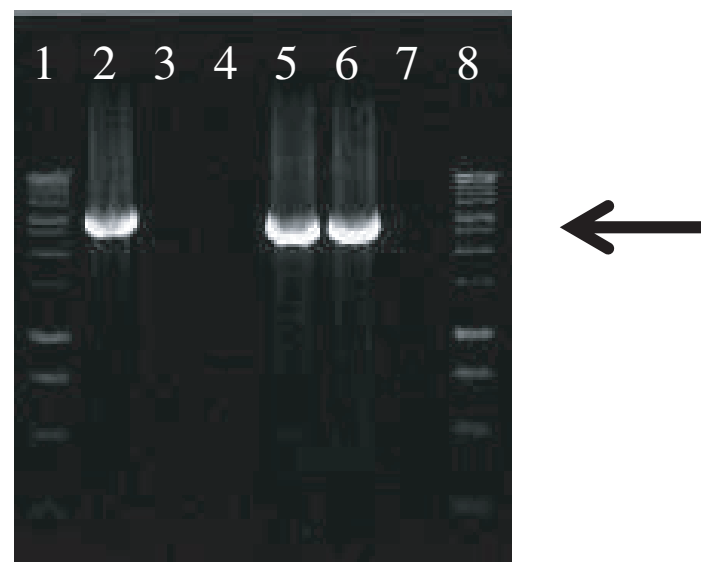

Figure 7. Detection of a CaMV gene VI-GFP PCR product. The CaMV gene VI-GFP PCR products $(5 \mu \mathrm{L})$ derived from amplification of DNAs using primers specific to the beginning of CaMV gene VI and to the GFP gene were analyzed after 40 cycles on a $1.5 \%$ agarose gel run at $100 \mathrm{~V}$ for $50 \mathrm{~min}$. Samples (1 and 8) $\mathrm{M}=$ (Promega) $1 \mathrm{kbp}$ molecular weight markers; (2-7) PCR products obtained from the following materials: (2) CaMV-GFP plasmid; (3) NonGFP OSR DNA; (4) 35S-GFP OSR DNA; (5) NonGFP OSR DNA plus CaMV-GFP plasmid; (6) 35S-GFP OSR DNA plus CaMV-GFP plasmid; (7) no template control. Arrow $=$ expected band of $2384 \mathrm{bp}$.

and the CaMV 35S RNA promoter present in each infected cell, integration, if it does occur, is at such a low frequency as to be undetectable by this approach.

\section{DISCUSSION}

Two questions concerning the use of the CaMV 35S RNA promoter to drive transgene expression have been addressed in this study: does its presence as part of a transgene in crop plants potentiate the integration of infecting CaMV to a significant degree, and/or could it be susceptible to silencing by infective CaMV? The results do not support the former contention, and indicate that the latter occurs only under limited circumstances.

Previous work concerning integration of CaMV into plant genomes did not detect any integration of the CaMV genome into the genome of its non-GM host plants to any measurable frequency (Gal et al., 1992; Schoelz and Wintermantel, 1993). No previously published study has assessed whether integration would occur into the genome of GM plants containing a CaMV 35S RNA promoter and that are hosts of CaMV. This study did not detect such integration in 944 samples taken from inoculated or systemically infected leaves, or from 2912 samples taken from the progeny of the inoculated plants. The lack of detection of CaMV integration does not demonstrate that CaMV is completely unable to integrate into plant genomes, but shows that such integration would have to occur at a level below our detection limit of one copy per 4000 haploid genomes or the equivalent of one in 2000 cells $(0.05 \%)$. Integration, if it occurred at or below this level would have virtually no consequences for the plant, because changes in gene expression that might occur as a result of integration in $0.05 \%$ of the cells of a population would not affect the plant as a whole and could not even be measured above background variation. Indeed, infection of $A$. thaliana by CaMV itself induced expression of 291 genes and reduced expression of 64 genes (our unpublished data), making possible effects superimposed by integration at a measurable frequency difficult to evaluate, let alone by integration at an extremely low frequency. Therefore, the above data indicate that the presence of the CaMV 35S RNA promoter does not lead to prolifigate integration into the plant genomes of its hosts.

Since CaMV is not considered to be seed transmissible (Hull, 1984), the absence of integrated virus in the progeny plants of infected seedlings may not be surprising. However, as it was unknown if the presence of the CaMV 35S RNA promoter would affect viral entry into germline tissues and allow integration, we examined this possibility further here. The progeny seedlings of the host plants of CaMV (A. thaliana, N. benthamiana and OSR) did not show any evidence of integration. Moreover, the progeny seedlings were not infected with CaMV, indicating that the presence of the CaMV 35S RNA promoter in the germline did not affect seed transmission of the virus itself.

The observation that gene silencing occurred in OSR for genes driven by the CaMV 35S RNA promoter, after infection by CaMV (Al-Kaff et al., 1998) was certainly 


\section{J. Squires et al.}

a cause for concern. This was modulated somewhat by the observation that CaMV-mediated gene silencing depended on the strain of CaMV (Al-Kaff et al., 2000) and on the host (Dale and Al-Kaff, 2006). In the work presented here, we have shown that a strain of CaMV that did not induce systemic silencing of transgene expression in OSR also did not do so in two other systemic hosts of CaMV (A. thaliana and N. benthamiana), as well as in plant species (tobacco) with only localized infection. Highly localized loss of fluorescence was observed in severely-infected $N$. benthamiana leaves, but this did not lead to the systemic silencing reported for OSR by Al-Kaff et al. (1998; 2000). In many of the plant species tested, loss of fluorescence did occur in the oldest leaves, but this occurred to the same extent in CaMV-infected and mock-inoculated leaves and probably represented a reduction of gene expression as a result of senescence (Mitsuhara et al., 1996).

Overall, our results complement those of Al-Kaff and colleagues and together these studies show that CaMVinfection of plants expressing transgenes regulated by the CaMV 35S RNA promoter will result in silencing of transgene expression only under limited circumstances, depending on the strain of the virus and the host species being evaluated. While some strains will silence gene expression in some host species, other strains probably will not silence gene expression in a range of host species. In the cases where the GM plant species are not systemic hosts for CaMV, it appears that infection by CaMV will not lead to transgene silencing, since silencing correlates with partial or complete recovery from virus infection (Al-Kaff et al., 1998). Therefore, in the case of OSR, the only cruciferous GM crop being grown in the field to date, the extent of transgene silencing likely to occur would be the product of the incidence of infection by CaMV and the frequency of occurrence in those fields of CaMV strains that can silence transgenes. As the distribution of transgene-silencing strains of CaMV in OSR growing countries is unknown, it is not possible to determine as such to what extent this potential silencing is a cause of concern. However, the lack of any reports of herbicide susceptibility arising in OSR from those countries that grow herbicide-resistant OSR, let alone those that can be attributed to silencing by CaMV infection, would suggest that this is not a case for general concern.

\section{MATERIALS AND METHODS}

\section{GM plants and propagation}

GM plants expressing the jellyfish GFP under the control of the CaMV 35S RNA promoter and the nos transcription termination signal were obtained from the following sources: A. thaliana ecotype C24 (Haseloff et al., 1997) was obtained from J. Haseloff (University of Cambridge); B. napus cv. Westar (Halfhill et al., 2001), and N. tabacum cv. Xanthi (Harper and Stewart, 2000) were obtained from C.N. Stewart, Jr. (Univ. of Tennessee); and N. benthamiana (Ruiz et al., 1998) was obtained from D.C. Baulcombe (Sainsbury Laboratory, Norwich, UK). All of these plants showed green fluorescence when viewed under either a hand-held UV lamp or a fluorescence microscope. While the transgene copy number was not ascertained directly, the genes were inherited as single copy genes.

The various GM plants were all propagated under license in a contained greenhouse maintained at $22 / 18{ }^{\circ} \mathrm{C}$ (day/night) with $16 \mathrm{~h}$ of daylight supplemented with artificial lighting at $250 \mu \mathrm{mol} \cdot \mathrm{m}^{-2} \cdot \mathrm{s}^{-1}$. Seeds were collected from non-inoculated and CaMV- infected GM plants and were propagated in soil as for the parental plants.

\section{Viruses: source and maintenance}

Since most of the experiments had to be done with a CaMV strain that could infect A. thaliana, B. napus and $N$. benthamiana, but was not aphid transmissible, so as to eliminate the risk of accidental natural transmission, the genetically engineered strain of CaMV designated H7 (Schoelz et al., 1986a) was chosen. Strain H7 is derived from the aphid-non-transmissible strain CM1841, but contains gene VI and part of the large intergenic region from the strain D4 that capable of infecting the above host species. CaMV strain D4 was shown to be mildly virulent on several plant species (Schoelz et al., 1986b) as well as A. thaliana ecotype Col-0 (Yu et al., 2003). The natural strain designated W260 (Gracia and Shepherd, 1985) was used to infect tobacco plants, since only this severe strain was known to produce a local infection in tobacco. Both CaMV strains were inoculated to $B$. rapa (turnip, cv. Just Right) and were maintained in this host in the contained greenhouse. Extracts of the CaMV-infected turnip plants were used to inoculate the various GM seedlings.

\section{Extraction of DNA and PCR analysis}

Plant DNA was isolated from plant tissues as described by Cullen et al. (2001) and the DNA was used for PCR analysis. To increase the chances of detecting integration, eight leaves of each of the $10 \mathrm{~A}$. thaliana plant were inoculated and each was extracted separately, while for the other plant species, one inoculated leaf or one systemically infected leaf of each of the 10 inoculated plants was sectored into eights, with each sector of a leaf extracted and analyzed separately. 
To detect the presence of the 35S RNA promoter, the two PCR primers were used, both able to bind to the 35S RNA promoter region: the forward primer was 5'-GCTCCTACAAATGCCATCA-3' (derived from nucleotide 7190-7208 of CaMV) and the reverse primer was 5'-GATAGTGGGATTGTGCGTCA-3' (complementary to nucleotide 7383-7365). The PCR reactions were done by denaturation at $94{ }^{\circ} \mathrm{C}$ for $3 \mathrm{~min}$, followed by 40 cycles of incubation at $94{ }^{\circ} \mathrm{C}$ for $1 \mathrm{~min}$, $54{ }^{\circ} \mathrm{C}$ for $1 \mathrm{~min}, 72^{\circ} \mathrm{C}$ for $1 \mathrm{~min}$, and a final incubation at $72{ }^{\circ} \mathrm{C}$ for $10 \mathrm{~min}$.

To detect integration of CaMV into the 35S RNA promoter, the forward primer was 5' GAGAACATAGAAAAACTCCTCAT-3' (corresponding to nucleotides 5779-5801) in CaMV gene VI, approximately $1400 \mathrm{bp}$ upstream of the CaMV 35S RNA promoter region), and the reverse primer was $5^{\prime}$ GGACACGCTGAACTTGTGGCCGTTTAC- ${ }^{\prime}$ ' (complementary to nucleotides $430-456$ of the GFP gene sequence). The PCR reactions were done by denaturation at $94{ }^{\circ} \mathrm{C}$ for $3 \mathrm{~min}$, followed by 35 cycles of incubation at $94{ }^{\circ} \mathrm{C}$ for $30 \mathrm{sec}, 50{ }^{\circ} \mathrm{C}$ for $30 \mathrm{sec}, 72{ }^{\circ} \mathrm{C}$ for $1.5 \mathrm{~min}$, and a final incubation at $72{ }^{\circ} \mathrm{C}$ for $10 \mathrm{~min}$. The primers used were described above. To confirm the ability of the primers to amplify a putative recombinant product under these conditions, CaMV-GFP, a virus with gene II replaced by the GFP gene, was used as the template. CaMV-GFP (Love et al., 2007) was obtained from J.J. Milner (Glasgow University).

\section{Detection and quantification of GFP fluorescence}

The inoculated and control GM plants were assessed for GFP accumulation at 10-day intervals by examination of the level of fluorescence of the GFP under a hand-held, Blak Ray long-wavelength UV lamp (UV Products) in the first instance. Fluorescence was measured using a stereomicroscope (Leica) fitted with a GFP3 filter, under UV light at $5 \times$ magnification and a Coolview camera capable of recording the fluorescence intensity. For A. thaliana, the top-most fully-expanded leaf was detached from each plant at each time point for microscopic visualization and measurement of fluorescence intensity. For the other three plant species, $1 \mathrm{~cm}$ squares were taken from the topmost fully-expanded leaf of each plant and used for visualization and fluorescence quantification. Images were analyzed with ImagePro software, using chip integration of 15 frames and a gain setting of 18 . The average intensity for all like plants was assessed at each time interval and the standard deviation of the fluorescence was calculated.

\section{ACKNOWLEDGEMENTS}

This work was funded by a grant from the UK Department of the Environment, Food and Rural Affairs. The authors thank Joel J. Milner for providing construct CaMVGFP, which was invented and constructed by Simon N. Covey and David S. Turner in the Department of Virus Research, John Innes Centre, Norwich, UK.

Received March 9, 2007; accepted August 29, 2007.

\section{REFERENCES}

Al-Kaff NS, Covey SN, Kreike MM, Page AM, Pinder R, Dale PJ (1998) Transcriptional and posttranscriptional gene silencing in response to a pathogen. Science 279: 2113-2115

Al-Kaff NS, Kreike MM, Covey SN, Pitcher R, Page AM, Dale PJ (2000) Plants rendered herbicide-susceptible by cauliflower mosaic virus-elicited suppression of a $35 \mathrm{~S}$ promoter-regulated transgene. Nature Biotechnol. 18: 995999

Assaad FF, Signer ER (1990) Cauliflower mosaic virus P35S promoter activity in Escherichia coli. Mol. Gen. Genet. 223: 517-520

Ballas N, Broido S, Soreq H, Loyter A (1989) Efficient functioning of plant promoters and poly(A) sites in Xenopus oocytes. Nucleic Acids Res. 17: 7891-7903

Baulcombe DC, Saunders GR, Bevan MW, Mayo MA, Harrison BD (1986) Expression of biologically active viral satellite RNA from the nuclear genome of transformed plants. Nature 321: 446-449

Bevan MW, Mason SE, Goelet P (1985) Expression of tobacco mosaic virus coat protein by the cauliflower mosaic virus promoter in plants transformed by Agrobacterium. EMBO J. 4: 1921-1926

Cullen DW, Lees AK, Toth IK, Duncan JM (2001) Conventional PCR and real-time quantitative PCR detection of Helminthosporium solani in soil and on potato tubers. Eur. J. Plant Pathol. 107: 387-398

Dale PJ, Al-Kaff NS (2006) http://www.defra.gov.uk/science/project_data/DocumentLibrary/CB02017/

CB02017_3425_FRP.doc

Franck A, Guilley H, Jonard G, Richards K, Hirth L (1980) Nucleotide sequence of cauliflower mosaic virus. Cell 21: 285-294

Gal S, Pisan B, Hohn T, Grimsley N, Hohn B (1992) Agroinfection of transgenic plants leads to viable cauliflower mosaic virus by intermolecular recombination. Virology 187 : $525-533$

Goldberg K-B, Kiernan J, Shepherd RJ (1991) A disease syndrome associated with expression of gene VI of caulimoviruses may be a nonhost reaction. Mol. PlantMicrobe Interact. 4: 182-189

Gracia O, Shepherd RJ (1985) Cauliflower mosaic virus in the nucleus of Nicotiana. Virology 146: 141-145 


\section{J. Squires et al.}

Guilley H, Dudley RK, Jonard G, Balázs E, Richards KE (1982) Transcription of cauliflower mosaic virus DNA: Detection of promoter sequences, and characterization of transcripts. Cell 30: 763-773

Halfhill MD, Richards HA, Mabon SA, Stewart Jr CN (2001) Expression of GFP and Bt genes in Brassica napus and hybridization with Brassica rapa. Theor. Appl. Genet. 103: 659-667

Harper BK, Stewart Jr CN (2000) Patterns of green fluorescent protein expression in transgenic plants. Plant Mol. Biol. Rep. 18: 121a-141i

Haseloff J, Siemering KR, Prasher DC, Hodge S (1997) Removal of a cryptic intron and subcellular localization of green fluorescent protein are required to mark transgenic Arabidopsis plants brightly. Proc. Natl. Acad. Sci USA 94: 2122-2127

Ho MW, Ryan A, Cummins J (1999) Cauliflower mosaic viral promoter - a recipe for disaster? Microb. Ecol. Health Dis. 11: 194-197

Ho MW, Ryan A, Cummins J (2000) Hazards of transgenic plants containing the cauliflower mosaic virus viral promoter. Microb. Ecol. Health Dis. 12: 6-11

Hull R (1984) Caulimovirus group. In CMI/AAB Description of Plant Viruses No. 295. Unwin Bros. Ltd., Old Woking, Surrey, UK

Hull R, Covey SN, Dale P (2000) Genetically modified plants and the 35S promoter: assessing the risks and enhancing the debate. Microb. Ecol. Health Dis. 12: 1-5

Jefferson RA, Kavanagh TA, Bevan MW (1987) GUS fusions: $\beta$-glucuronidase as a sensitive and versatile gene fusion marker in higher plants. EMBO J. 6: 3901-3907

Love AJ, Laval V, Geri C, Laird J, Tomos AD, Hooks MA, Milner JJ (2007) Components of Arabidopsis defenseand ethylene-signaling pathways regulate susceptibility to Cauliflower mosaic virus by restricting long-distance movement. Mol. Plant-Microbe Interact. 20: 659-670

Mitsuhara I, Ugaki M, Hirochika H, Ohshima M, Murakami T, Gotoh Y, Katayose Y, Nakamura S, Honkura R, Nishimiya S, Ueno K, Mochizuki A, Tanimoto H, Tsugawa H, Otsuki Y, Ohashi Y (1996) Efficient promoter cassettes for enhanced expression of foreign genes in dicotyledonous and monocotyledonous plants. Plant Cell Physiol. 37: 49-59

Morel JB, Tepfer M (2000) Pour une évaluation scientifique des risques : le cas du promoteur 35S. Biofutur 201: 32-35

Odell JT, Nagy F, Chua NH (1985) Identification of DNA sequences required for activity of the cauliflower mosaic virus 35S promoter. Nature 313: 810-812
Ow DW, Jacobs JD, Howell SH (1987) Functional regions of the cauliflower mosaic virus 35S RNA promoter determined by the use of the firefly luciferase gene as a reporter of promoter activity. Proc. Natl. Acad. Sci. USA 84: 4870-4874

Pobjecky N, Rosenberg GH, Dinter-Gottlieb G, Kaufer D (1990) Expression of the beta-glucuronidase gene under the control of the CaMV 35S promoter in Schizosacharomyces pombe. Mol. Gen. Genet. 220: 314-316

Ruiz MT, Voinnet O, Baulcombe DC (1998) Initiation and maintenance of virus-induced gene silencing. Plant Cell 10: 937-946

Rüth J, Hirt H, Schweyen RJ (1992) The cauliflower mosaic virus $35 \mathrm{~S}$ promoter is regulated by cAMP in Saccharomyces cerevisiae. Mol. Gen. Genet. 235: 365-372

Rüth J, Schweyen RJ, Hirt H (1994) The plant transcription factor TGA1 stimulates expression of the CaMV 35S promoter in Saccharomyces cerevisiae. Plant Mol. Biol. 25: 323328

Schoelz JE (2006) Viral determinants of resistance versus susceptibility. In Loebenstein G, Carr JP, eds, Natural Resistance Mechanisms of Plants to Viruses, Springer, The Netherlands, pp 13-33

Schoelz JE, Wintermantel WM (1993) Expansion of viral host range through complementation and recombination in transgenic plants. Plant Cell 5: 1669-1679

Schoelz JE, Shepherd RJ, Daubert SD (1986a) Region VI of cauliflower mosaic virus encodes a host range determinant. $J$. Mol. Cell Biol. 6: 2632-2637

Schoelz JE, Shepherd RJ, Richins DR (1986b) Properties of an unusual strain of cauliflower mosaic virus. Phytopathology 76: $451-454$

Sun L, Cai H, Xu W, Hu Y, Lin Z (2002) CaMV 35S promoter directs beta-glucuronidase expression in Gonoderma lucidum and Pleurotus citrinopileatus. Mol. Biotechnol. 20: 239-244

Tepfer M, Gaubert S, Leroux-Coyau M, Prince S, Houdebine LM (2004) Transient expression in mammalian cells of transgenes transcribed from the Cauliflower mosaic virus 35S promoter. Environ. Biosafety Res. 3: 91-97

Vlasák J, Šmahel M, Pavlik A, Pavingerová D, Břiza J (2003) Comparison of hCMV intermediate early and CaMV $35 \mathrm{~S}$ promoters in both plant and human cells. J. Biotechnol. 103: 197-202

Yu W, Murfett J, Schoelz JE (2003) Differential induction of symptoms in Arabidopsis by P6 of Cauliflower mosaic virus. Mol. Plant-Microbe Interact. 16: 35-42 